Contrib. Plasma Phys. 41 (2001) 2-3, 135-138

\title{
Path Integral Simulations of the Thermodynamic Properties of Quantum Dense Plasma
}

\author{
V.S. Filinovi), M. Bonitz ${ }^{2)}$, D. Kremp ${ }^{2)}$, W.-D. Kraefr ${ }^{3)}$, \\ W. Ebeling ${ }^{4}$, P.R. Levashovi), V.E. Fortov ${ }^{1)}$ \\ ${ }^{1)}$ High Energy Density Research Center Rus.Ac.Sc., Izhorskaya street 13-19, \\ Moscow 127412, Russia \\ ${ }^{2)}$ Fachbereich Physik, Univ. Rostock, Universitätspl. 3, D-18051 Rostock \\ 3) Institut für Physik der Univ. Greifswald, Domstr. 10a, D-17489 Greifswald \\ ${ }^{4)}$ Inst. für Physik, Humboldt-Univ. Berlin, Invalidenstr. 110, D-10115 Berlin
}

Received 26 September 2000

\begin{abstract}
A novel path integral representation of the many-particle density operator is presented which makes direct Fermionic path integral Monte Carlo simulations feasible over a wide range of parameters. The method is applied to compute the energy of a dense hydrogen plasma in the region of intermediate coupling and degeneracy and is compared to analytical and experimental results.

Many interesting phenomena in dense plasmas occur in situations where Coulomb and quantum effects are important simultaneously. Among the most promising theoretical approaches to these systems are path integral quantum Monte Carlo (PIMC) techniques. However, for Fermi systems, they are hampered by the so-called sign problem which lead to the incorporation of additional assumptions (restricted PIMC concept [1]). In this work we demonstrate that, for many current problems in dense warm plasmas $\left(k_{B} T>0.1 R y\right)$, rigorous direct PIMC simulations can be carried out with acceptable efficiency. Here, we report results for the internal energy of partially ionized hydrogen over a broad range of coupling and degeneracy parameters, $\Gamma=\left(4 \pi n_{e} / 3\right)^{1 / 3} e^{2} / 4 \pi \epsilon_{0} k_{B} T$ and $\chi=n_{e} \lambda_{e}^{3}\left[\lambda_{e}\right.$ is the electron thermal wave length $\left.\lambda_{e}^{2}=2 \pi \hbar^{2} \beta / m_{e}\right]$.

Thermodynamic quantities are computed from the partition function which, for a binary mixture of $N_{e}$ electrons and $N_{i}$ protons is conveniently written as [2]

$$
Z\left(N_{e}, N_{i}, V, \beta\right)=\frac{Q\left(N_{e}, N_{i}, \beta\right)}{N_{e} ! N_{i} !}, \quad Q\left(N_{e}, N_{i}, \beta\right)=\sum_{\sigma} \int_{V} d q d r \rho(q, r, \sigma ; \beta) .
$$

Here, $q \equiv\left\{\mathbf{q}_{1}, \mathbf{q}_{2}, \ldots, \mathbf{q}_{N_{i}}\right\}$ comprises the coordinates of the protons, and $\sigma=$ $\left\{\sigma_{1}, \ldots, \sigma_{N_{e}}\right\}$ and $r \equiv\left\{\mathbf{r}_{1}, \ldots, \mathbf{r}_{N_{e}}\right\}$ the electron spins and coordinates, respectively. The density matrix $\rho$ in Eq. (1) is represented in standard way by a path integral [3]

$$
\begin{aligned}
& \rho(q, r, \sigma ; \beta)=\frac{1}{\lambda_{i}^{3 N_{i}} \lambda_{\Delta}^{3 N_{e}}} \sum_{P}( \pm 1)^{\kappa_{P}} \int_{V} d r^{(1)} \ldots d r^{(n)} \\
& \times \rho\left(q, r, r^{(1)} ; \Delta \beta\right) \ldots \rho\left(q, r^{(n)}, \hat{P} r^{(n+1)} ; \Delta \beta\right) \mathcal{S}\left(\sigma, \hat{P} \sigma^{\prime}\right),
\end{aligned}
$$
\end{abstract}

where $\Delta \beta \equiv \beta /(n+1), \lambda_{\Delta}^{2}=2 \pi \hbar^{2} \Delta \beta / m_{e}, r^{(n+1)} \equiv r$ and $\sigma^{\prime} \equiv \sigma$. The electron spin gives rise to the spin part of the density matrix $\mathcal{S}$, whereas exchange effects are 
accounted for by the permutation operator $\hat{P}$ and the sum over the permutations with parity $\kappa_{P}$. Following Refs. [4, 2], we use a modified representation (3) of the high-temperature density matrices on the r.h.s. of Eq. (2)

$$
\begin{array}{r}
\sum_{\sigma} \rho(q, r, \sigma ; \beta)=\frac{1}{\lambda_{i}^{3 N_{i}} \lambda_{\Delta}^{3 N_{e}}} \sum_{s=0}^{N_{e}} \rho_{s}(q,[r], \beta), \\
\rho_{s}(q,[r], \beta)=\frac{C_{N_{e}}^{s}}{2^{N_{e}}} e^{-\beta U(q,[r], \beta)} \prod_{l=1}^{n} \prod_{p=1}^{N_{e}} \phi_{p p}^{l} \operatorname{det}\left|\psi_{a b}^{n, 1}\right|_{s}, \\
U(q,[r], \beta)=U^{i}(q)+\sum_{l=0}^{n} \frac{U_{l}^{e}([r], \beta)+U_{l}^{e i}(q,[r], \beta)}{n+1},
\end{array}
$$

which is suitable for efficient direct fermionic PIMC simulations of plasmas. The error of Eq. (3) is of the order $(\beta R y)^{2} \chi /(n+1)$ and vanishes with growing number of beads. Further, $U^{i}, U_{l}^{e}$ and $U_{l}^{e i}$ denote the sum of the binary interaction potentials $\Phi^{a b}$ between protons, electrons at vertex "l" and electrons (vertex "l") and protons, respectively, for which the Kelbg potential [5] was found to be a very good approximation [6].

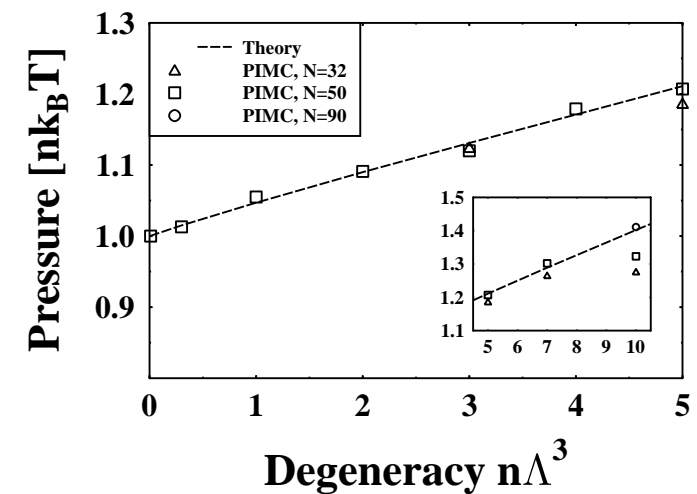

Fig. 1: Pressure of an ideal plasma of degenerate electrons and classical protons. PIMC simulation results with varying particle number are shown: $N=32$ (triangles), $N=50$ (squares) and $N=90$ (circles) are compared to the exact analytical result (dashed line).

strate our numerical scheme for a two-component electron-proton plasma. In the simulations we used $N_{e}=N_{p}=50$ and $n=6 \ldots 20$. To test the MC procedure we first consider a mixture of ideal electrons and protons for which the thermodynamic quantities are known analytically, e.g. [7]. Fig. 1 shows our numerical results for the pressure together with the theoretical curve. The agreement, up to values of the degeneracy parameter $\chi$ as large as 10 is evident and improves with increasing particle number. This clearly proofs that our method correctly samples the fermionic permutations. Furthermore, we note that our scheme is numerically efficient which is primarily due to the representation (3) and the determinant (4) allowing for fast generation of a $\mathrm{MC}$ sequence of $\mathrm{N}$-particle configurations. In particular, the acceptance probability of new configurations is proportional to the absolute value of the ratio of the exchange 
determinants of two subsequent configurations, while the sign of the determinants is included into the weight function of each configuration.

Let us now turn to the case of interacting electrons and protons. We have performed a series of calculations in which the classical coupling parameter $\Gamma$ was kept constant while the degeneracy parameter $\chi$ was varied. For definiteness, the comparison is restricted to a H-plasma in the density-temperature region with the following borders $0.2<\Gamma<1.6$ and $0.25<n_{e} \Lambda_{e}^{3}<5$ which may be called "rhombus of moderate nonideality degeneracy" [6]. With respect to analytical treatment this region is of particular difficulty since none of the known limiting expressions is valid. The results of our calculations are given in Fig. 2. A very interesting result is that the energy curves in Fig. 2 become almost parallel to each other as the degeneracy increases.

In contrast, for $\Gamma>0.6$, reduction of the degeneracy below $\chi \sim 1$ leads to a rapid decrease of the energy which is due to the formation of atoms and molecules which is also clearly seen in the pair distribution functions, cf. [2]. Our analyis shows that, for weak coupling (cf. $\quad \Gamma \leq 0.4$ ) and small degeneracy parameters, $\chi<0.5$, there is good agreement with analytical theories [2], however, with increasing $\chi$ and $\Gamma$, the deviations are growing rapidly. This figure also contains data points of recent restricted path integral calculations of Militzer et al. [1] (cf. the large triangles) compared to our results (large squares) for values of the coupling parameter in the range of $0.17 \ldots 1.35$. Evidently, the agreement is very good. A more detailed comparison is given in Ref. [6].

Finally, we have included in Fig. 2 results of improved Padé approximations. They are analytically exact up to quadratic terms in the density and interpolate between the virial expansions and the highdensity asymptotic expressions

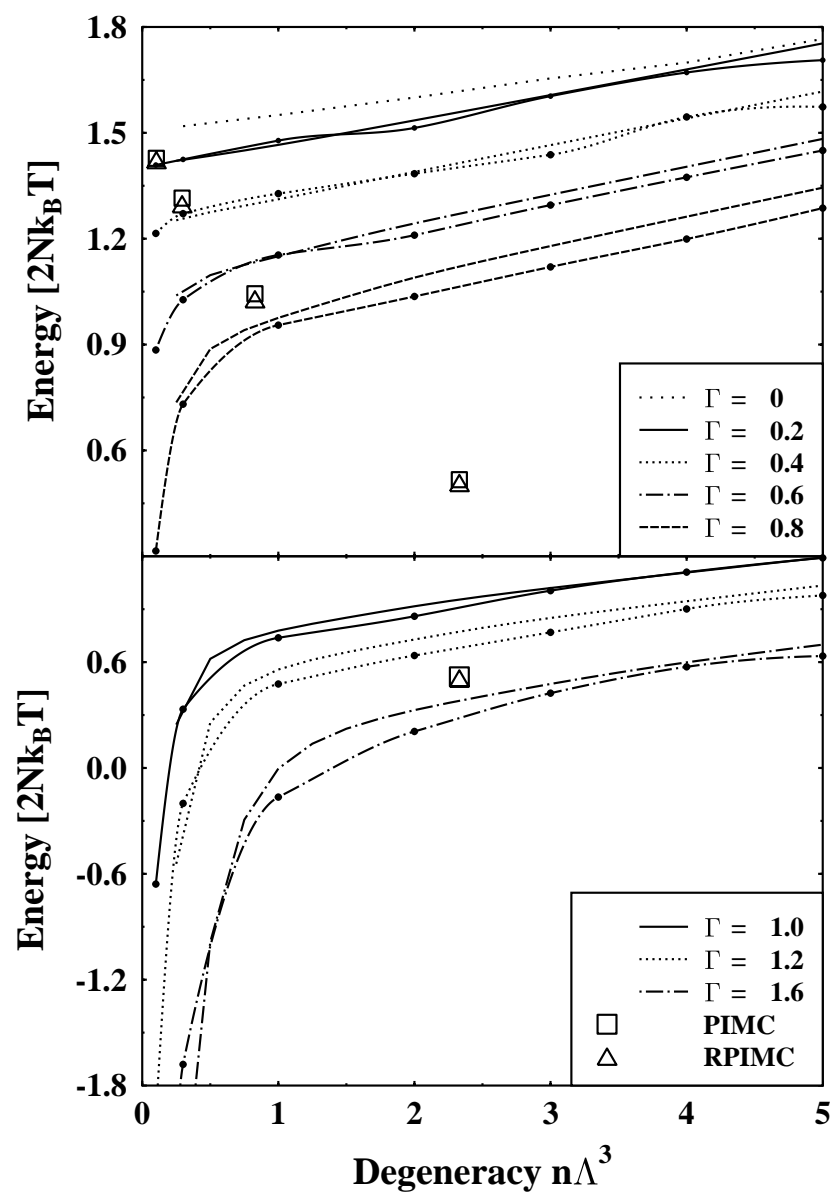

Fig. 2: Comparison of Padé calculations (lines without symbols) of the internal energy with the direct PIMC results (lines with full circles). Also shown are data points from restricted PIMC simulations [1] vs. our simulation results for 4 values of $\Gamma$ : from top to bottom: $\Gamma=0.169,0.338,0.672,1.35$. (The last point appears on both figure parts.)

and also include bound states by using the chemical picture [6]. Obviously, these formulas fit the simulation data rather well, demonstrating the usefulness of this approach. 
Fig. 3 shows our results for the total energy of hydrogen for a constant temperature, $T=125,000 K$, where the degree of ionization is large. Again, a comparison with RPIMC data is given. Further, analytical results from quantum statistical calculations are shown which include Hartree-Fock and Montroll-Ward diagrams [7].

The agreement up to densities of the order $10^{23} \mathrm{~cm}^{-3}$ is rather good. However, in the region of the Mott transition, around $10^{24} \mathrm{~cm}^{-3}$, the analytical model, as expected, underestimates the Coulomb interaction. On the other hand, they are known to give the correct asymptotics for the strongly degenerate plasma at very high densities which will be explored in more detail elsewhere [8].

Based on extensive investigations, we may conclude that our direct PIMC simulations provide accurate results for dense plasmas at $T>0.1 R y$ and for densities up to the Mott point where no reli-

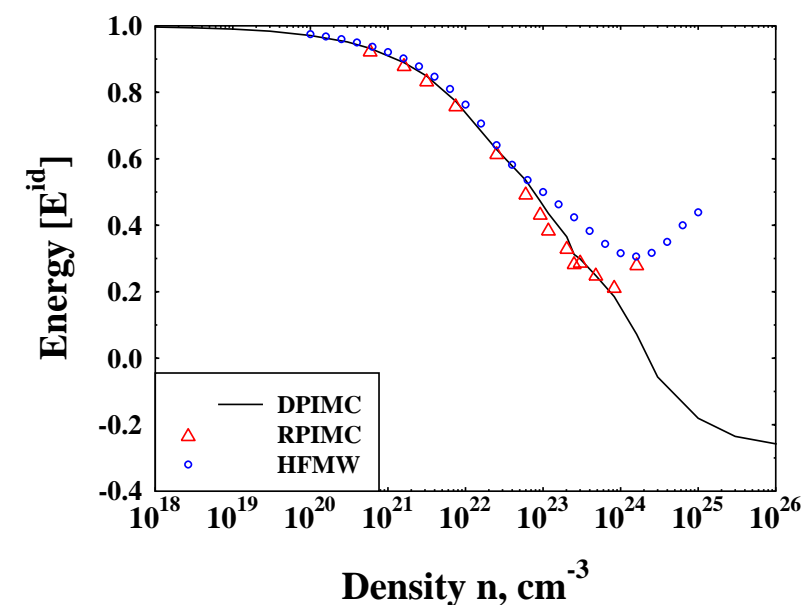

Fig. 3: Energy of dense hydrogen at $T=125,000 \mathrm{~K}$ computed analytically (Hartree-Fock plus MontrollWard terms [7]) and from restricted [1] and direct PIMC results.

able theoretical models exist. The data for densities beyond $10^{25} \mathrm{~cm}^{-3}$ are preliminary as there more fermionic beads and further improvements of the high temperature density matrix may be needed.

\section{Acknowledgements}

This work is supported by the Deutsche Forschungsgemeinschaft (MercatorProgramm) and the NIC Jülich. We acknowledge stimulating discussions with M. Schlanges and thank B. Militzer for providing the data of Ref. [1].

\section{References}

[1] B. Militzer, and D. Ceperley, Phys. Rev. Lett. 85, 1890 (2000).

[2] V.S. Filinov, M. Bonitz, and V.E. Fortov, JETP Letters 72, 361 (2000); V.S. Filinov, M. Bonitz, D. Kremp, and V.E. Fortov, Phys. Lett. A, accepted

[3] R.P. Feynman, and A.R. Hibbs, Quantum mechanics and path integrals, McGraw-Hill, New York 1965

[4] V.S. Filinov, High Temperature 13, 1065 (1975) and 14, 225 (1976); B.V. Zelener, G.E. Norman, and V.S. Filinov, High Temperature 13, 650 (1975)

[5] See G. Kelbg, Ann. Physik, 12, 219 (1963); 13, 354; 14, 394 (1964); W. Ebeling, H.J. Hoffmann, and G. Kelbg, Beitr. Plasmaphys. 7, 233 (1967) and references therein.

[6] V.S. Filinov, M. Bonitz, W. Ebeling, and V.E. Fortov, subm. to Plasma Phys. Cont. Fusion

[7] W.D. Kraeft, D. Kremp, W. Ebeling, and G. Röpke, Quantum Statistics of Charged Particle Systems, Akademie-Verlag Berlin 1986.

[8] V.S. Filinov, M. Bonitz, W.D. Kraeft, D. Kremp, and V.E. Fortov, to be published. 